Thorax (1974), 29, 248.

\title{
Multiple thoracotomy for metastatic pulmonary neoplasm
}

\author{
PETER M A R KS \\ Westminster Hospital, Horseferry Road, London SWI
}

\begin{abstract}
Marks, P. (1974). Thorax, 29, 248-251. Multiple thoracotomy for metastatic pulmonary neoplasm. Surgical excision of solitary, metastatic, malignant lesions of the lung is reported as of benefit in certain cases in which the primary tumour apparently has been completely removed and in which there is no evidence of further metastatic spread. It has been shown that in a few instances survival for many years may be expected to follow such a procedure, while at other times the degree of palliation of pulmonary symptoms makes the operation worthwhile.

Two patients who had metastatic lung deposits successfully removed are described. Further pulmonary metastases, without evidence of metastases elsewhere or recurrence of the original lesion, were resected. In both cases remarkable palliation of symptoms was afforded and both are alive at the time of writing (1 November 1973).
\end{abstract}

\section{CASE REPORTS}

PATIENT 1 A man aged 49 presented in January 1961 with a tumour mass in the lower end of the right femur. He was treated by radiotherapy and subsequent hip disarticulation. Prophylactic radiotherapy to the chest was also given. Histology showed spindlecell proliferation destroying bone and a diagnosis of osteogenic sarcoma was made. He remained well and free from recurrence until September 1969 when a mass was disclosed in the right lower lobe of the lung (Fig. 1). He underwent right thoracotomy and wedge excision of the tumour. Postoperative recovery was uneventful.

At review in March 1971 he was found to have a solitary metastasis $1.5 \mathrm{~cm}$ in diameter in the right mid-pulmonary zone (Fig. 2). He was readmitted for operation. At thoracotomy two nodules were found in the right lower lobe presenting on the surface, one near the fissure and the smaller one on the diaphragmatic surface of the lobe. Both nodules were resected by wedge resection. The patient made a good postoperative recovery. At review in November 1971 he was noted to have an elongated firm mass in the line of the rib below the thoracotomy scar adjacent to the drainage wound. At operation one week later excision of this area of chest wall was carried out. Histology showed metastatic osteogenic sarcoma. The wound did not heal satisfactorily and he developed a persistent discharging sinus. Three months later a chest radiograph revealed a $3 \mathrm{~cm}$ nodule in the right lower lobe posteriorly.

In view of the distressing symptoms caused by the sinus operation was undertaken in March 1972. This revealed localized neoplastic involvement of both the sinus and chest wall in continuity with the lung, and $\mathbb{\odot}$ a further secondary neoplastic deposit in the anterior segment of the upper lobe. Both lesions were resected separately with total wide resection of the chest wall lesion and pulmonary wedge resection of the lesion in the residual right lower lobe. Despite the extensive nature of the surgery the patient made an uneventful recovery and was discharged two weeks after admission. At the time of writing (1 November 1973) the patient is alive and well and free from all signs of recurrence.

PATIENT 2 In January 1966 this male patient was aged 19 at the time of diagnosis of a malignant 0 teratoma of the right testis. There were no pulmonary or hepatic metastases. Orchidectomy with ligation of $\frac{7}{0}$ the cord was performed. Histological examination of the specimen showed this to be a malignant teratoma $N$ (intermediate type $\mathrm{A}$ ). $\mathrm{He}$ received radiotherapy to anterior and posterior pelvis and para-aortic region. In addition he received $2,500 \mathrm{R}$ to the chest prophylactic- $\tilde{\omega}$ ally, and actinomycin $\mathrm{D}$. He made an uneventful recovery and was followed up.

In April 1967 tomography revealed a metastasis in each lung field in the mid-zones (Fig. 3). Clinically of the patient had pain in the chest and malaise. Bilateral thoracotomies were performed. An isolated nodule, $3 \mathrm{~cm}$ in diameter, was found in the anterior border of the left upper lobe. Wedge resection was performed. On the right a nodule, $4 \mathrm{~cm}$ in diameter, in the anterior segment of the right upper lobe was found. This also was removed by wedge resection. 


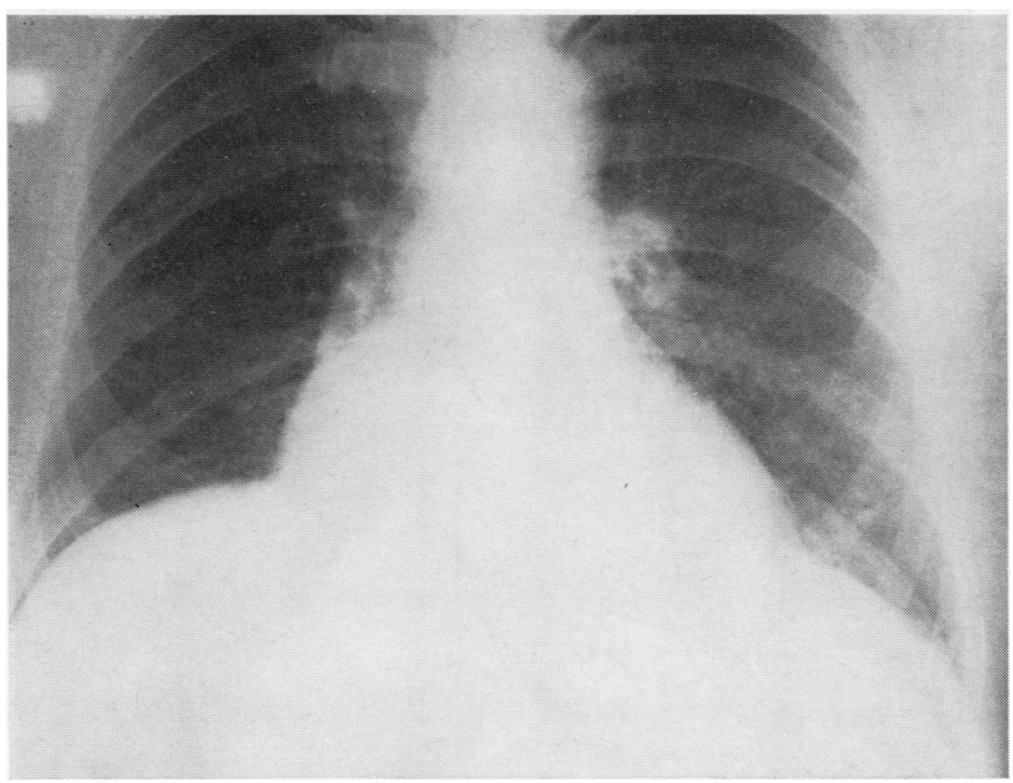

FIG. 1. Patient 1. (September 1969) Mass in right lower zone behind right border of heart.

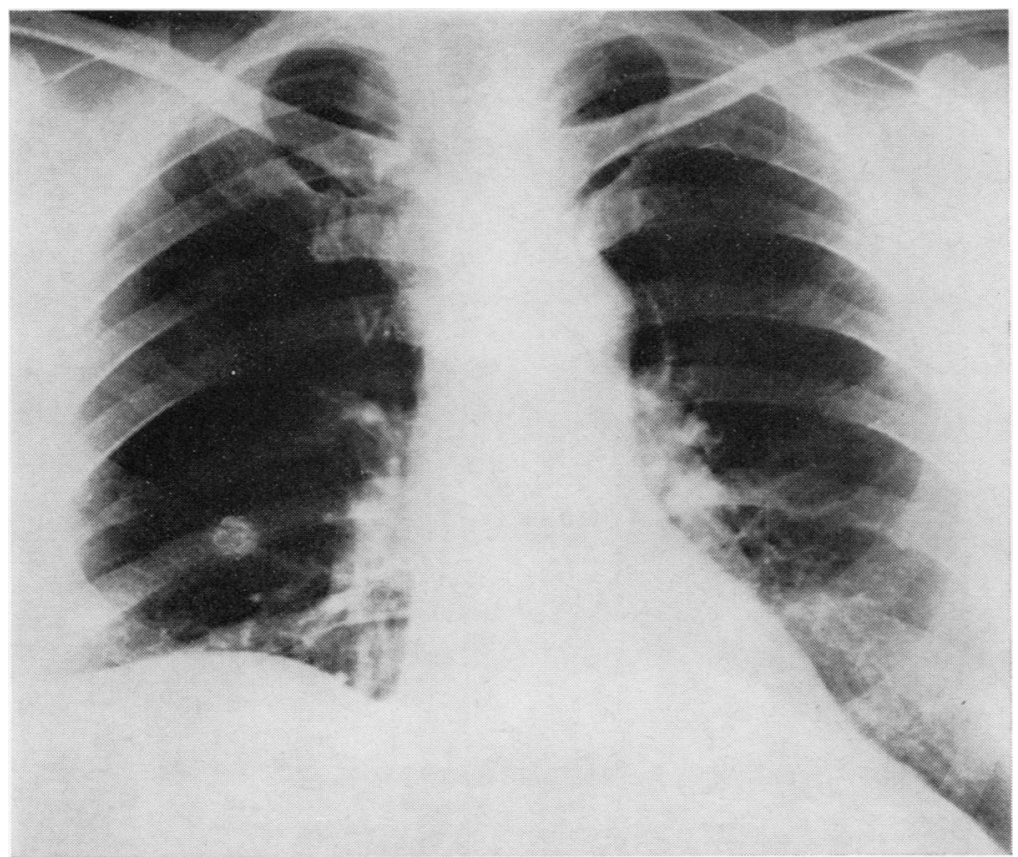

FIG. 2. Patient 1. (March 1971) Mass in right mid-pulmonary zone. 


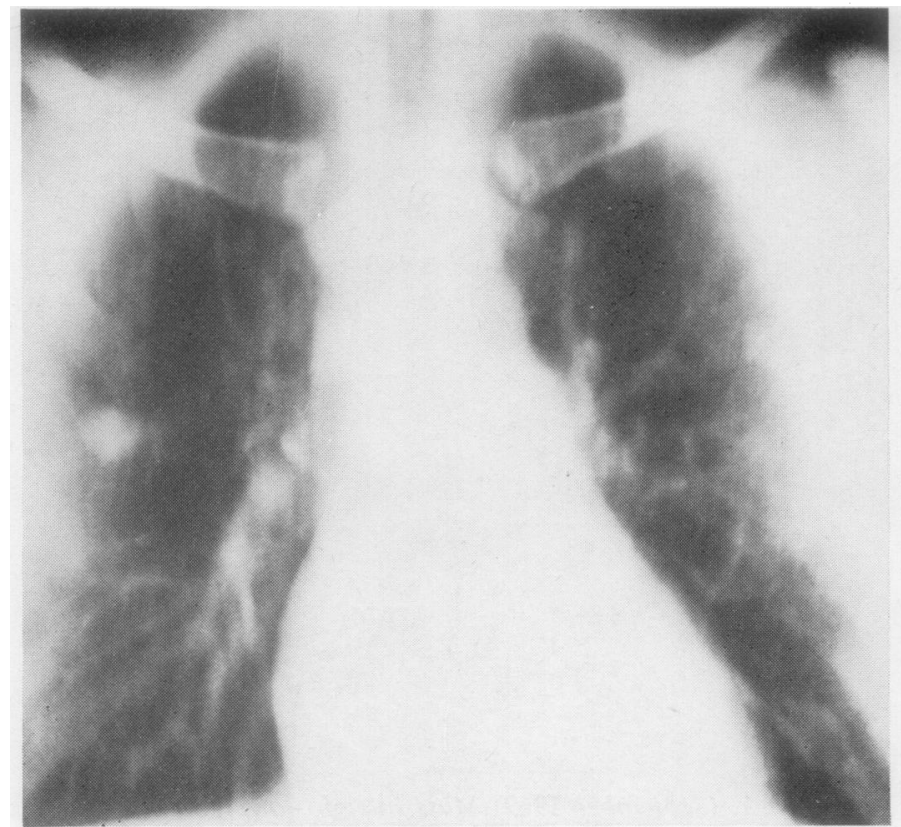

FIG. 3. Patient 2. (A pril 1967) One metastasis shown in right midzone, and one shown indistinctly in left mid-zone in tomogram.

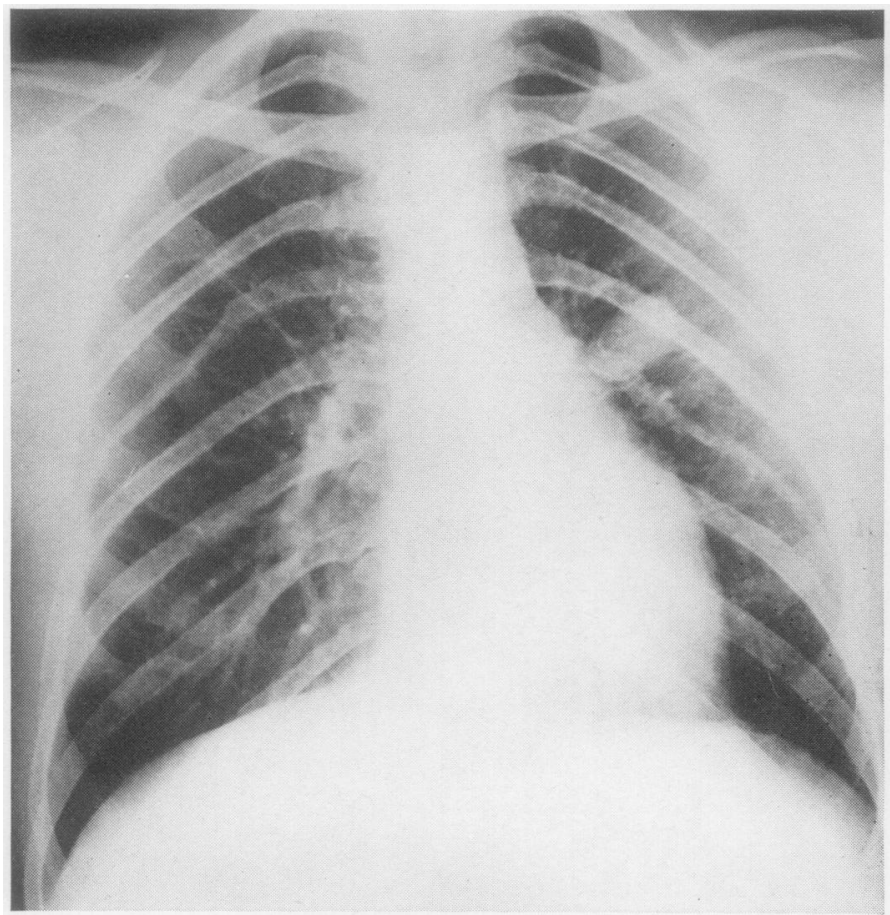

FIG. 4. Patient 2. (November 1968) Opacity shown in left mid-zone. 
Histology showed these deposits to be teratomas with differentiation similar to the primary lesion. The patient made a good recovery and was discharged home two weeks later.

A routine chest radiograph 18 months later showed a moderately sized oval-shaped opacity in the left midzone (Fig. 4). A left thoracotomy was performed through the bed of the sixth rib and a deposit, $4 \mathrm{~cm}$ in diameter, was found close to the left upper lobe hilum; there was no spread across the fissure or to the chest wall. Left upper lobectomy was performed. Histology confirmed that this was a metastasis from a teratoma. The patient made a good recovery after operation and was discharged home three weeks later (28 November 1968). Subsequent follow-up to the present day (1 November 1973) has revealed no further metastases and the patient has remained in good health.

\section{DISCUSSION}

Seiler, Claggett, and McDonald's (1950) series reviewed the surgical treatment of metastatic pulmonary neoplasms with an analysis of 62 cases. Their conclusion was that palliative pulmonary resection for metastatic malignant lesions seemed justifiable on the basis of prolongation of life and the degree of comfort afforded to the patient. Drew (1961) concluded that surgery was justifiable and reported several long survivals. Sellors (1970) concluded that excision of a single or isolated group of secondaries is not necessarily followed by further metastases.
Two cases are reported in which removal of metastatic pulmonary deposits resulted in striking alleviation of distressing pulmonary symptoms. One patient survived seven-and-a-half years after removal of the primary teratoma and six-and-ahalf years from removal of the first metastasis, and the other $12 \frac{1}{2}$ years after removal of the primary osteogenic sarcoma and four years from removal of the first metastasis. Both patients underwent multiple thoracotomy. In each patient the original diagnosis was histologically proven and the nature of the secondary deposit was similar.

I am grateful to Mr. Peter Jones, M.V.O., F.R.C.S., thoracic surgeon at Westminster Hospital, for permission to report these cases.

\section{REFERENCES}

Drew, C. (1961). Pulmonary resection for metastases. Progress in Clinical Surgery, edited by R. Smith, series II, p. 310. Churchill, London.

Seiler, H. H., Claggett, O. T., and McDonald, J. R. (1950). Pulmonary resection for metastatic malignant lesions. Journal of Thoracic Surgery, 19, 655.

Sellors, T. H. (1970). Treatment of isolated pulmonary metastases. British Medical Journal, 2, 253.

Requests for reprints to: Dr. P. Marks, Coronary Unit, Westminster Hospital, Horseferry Road, London SW1. 